\title{
MEASURE OF NON-COMPACTNESS AND INTERPOLATION METHODS ASSOCIATED TO POLYGONS
}

\author{
FERNANDO COBOS \\ Departamento de Análisis Matemático, Facultad de Matemáticas, Universidad Complutense de Madrid, \\ 28040 Madrid, Spain \\ e-mail: cobos@eucmax.sim.ucm.es \\ PEDRO FERNÁNDEZ-MARTÍNEZ \\ Departamento de Matemática Aplicada, Facultad de Informática, Universidad de Murcia, Campus de \\ Espinardo, 30071 Espinardo (Murcia), Spain \\ e-mail: pedrofdz@fcu.um.es \\ ANTÓN MARTÍNEZ \\ Departamento de Matemática Aplicada, Escuela Técnica Superior de Ingenieros Industriales, Universidad de \\ Vigo, Lagoas-Marcosende, 36200 Vigo, Spain \\ e-mail: antonmar@uvigo.es
}

(Received 13 March, 1997)

\begin{abstract}
We establish an estimate for the measure of non-compactness of an interpolated operator acting from a $J$-space into a $K$-space. Our result refers to general Banach $N$-tuples. We also derive estimates for entropy numbers if some of the $N$-tuples reduce to a single Banach space.
\end{abstract}

0. Introduction. The investigation of the behaviour of compactness under interpolation methods for $N$-tuples of Banach spaces associated with polygons was started by Cobos and Peetre in [8]. There they studied the case when the interpolated operator acts between two $K$-spaces or two $J$-spaces. Later, Cobos, Kühn and Schonbek [7] continued this research by considering operators acting from a $J$-space into a $K$-space. Optimality of all these results was analyzed in [3].

It is natural to investigate now how far from being compact an interpolated operator can be, a question that was already considered by Edmunds and Teixeira [12] and by the present authors [5] in the case of the real method for couples, and by Nikolova [10] in the present context of $N$-tuples of Banach spaces. Nikolova derived estimates for the measure of non-compactness of an interpolated operator provided that one of the $N$-tuples degenerates into a single Banach space or that the image $N$ tuple satisfies a certain approximation condition.

We deal here with general $N$-tuples, without requiring any approximation hypothesis, and we establish an estimate for the measure of non-compactness when the interpolated operator $T$ acts from a $J$-space into a $K$-space. In the special situation where one of the restrictions of $T$ is compact, we recover the compactness result of Cobos, Kühn and Schonbek [7].

Our techniques are based on some ideas introduced in [7] that allow us to use efficiently the information known for the real interpolation method for couples. The relevant estimate in this last case was derived by the authors in [5].

The organization of the paper is as follows. In Section 1 we recall some basic facts on measure of non-compactness and on methods associated with polygons. Section 2 contains the estimate for the measure of non-compactness. Finally, in Section 3 , we study degenerate cases when one of the $N$-tuples reduces to a single 
Banach space. In these special cases we show estimates for entropy numbers that improve Nikolova's results mentioned before.

1. Preliminaries Let $A$ and $B$ be Banach spaces and $T \in \mathcal{L}(A, B)$ be a bounded linear operator acting from $A$ into $B$. The $n$-th entropy number $e_{n}(T)$ of $T$ is defined as the infimum for all $r>0$ such that there are $b_{1}, \ldots, b_{m} \in B$ with $m \leq 2^{n-1}$ and

$$
T\left(\mathcal{U}_{A}\right) \subseteq \bigcup_{j=1}^{m}\left\{b_{j}+r \mathcal{U}_{B}\right\}
$$

Here $\mathcal{U}_{A}$ and $\mathcal{U}_{B}$ are the closed unit balls of $A$ and $B$ respectively. The measure of non-compactness $\beta(T)$ of $T$ is defined as the infimum of all $r>0$ such that there exists a finite number of elements $b_{1}, \ldots, b_{s} \in B$ so that

$$
T\left(\mathcal{U}_{A}\right) \subseteq \bigcup_{j=1}^{s}\left\{b_{j}+r \mathcal{U}_{B}\right\}
$$

Clearly $\|T\|=e_{1}(T) \geq e_{2}(T) \geq \cdots \geq 0$, and $e_{n}(T) \rightarrow \beta(T)$ as $n \rightarrow \infty$. Also $\beta(T)=0$ if and only if $T$ is compact. We refer to [2], [9] and [11] for others properties of these notions.

Let $\Pi=\overline{P_{1} \ldots P_{N}}$ be a convex polygon in the affine plane $\mathrm{R}^{2}$ with vertices $P_{j}=\left(x_{j}, y_{j}\right),(j=1, \ldots, N)$. Let $\bar{A}=\left\{A_{1}, \ldots, A_{N}\right\}$ be a Banach $N$-tuple, that is to say, a family of $N$ Banach spaces $A_{j}$ all of them continuously embedded in a common linear Hausdorff space. In what follows, it will be useful to imagine each space of the $N$-tuple $\bar{A}$ as sitting on the vertex $P_{j}$.

By means of the polygon $\Pi$, we define the following family of norms in the sum $\Sigma(\bar{A})=A_{1}+\cdots+A_{N}$ :

$$
K(t, s ; a)=K(t, s ; a ; \bar{A})=\inf \left\{\sum_{j=1}^{N} t^{x_{j}} s^{y_{j}}\left\|a_{j}\right\|_{A_{j}}: a=\sum_{j=1}^{N} a_{j}, a_{j} \in A_{j}\right\} t, s>0 .
$$

Similarly, in $\Delta(\bar{A})=A_{1} \cap \cdots \cap A_{N}$, we consider the family of norms

$$
J(t, s ; a)=J(t, s ; a ; \bar{A})=\max _{1 \leq j \leq N}\left\{t^{x_{j}} s^{y_{j}}\|a\|_{A_{j}}\right\} .
$$

Given $(\alpha, \beta)$ in the interior of $\Pi,(\alpha, \beta) \in \operatorname{Int} \Pi$, and $1 \leq q \leq \infty$, the $K$-interpolation space $\bar{A}_{(\alpha, \beta), q ; K}$ is formed by all elements $a \in \Sigma(\bar{A})$ for which the norm

$$
\|a\|_{(\alpha, \beta), q ; K}=\left(\sum_{(m, n) \in Z^{2}}\left(2^{-\alpha m-\beta n} K\left(2^{m}, 2^{n} ; a\right)\right)^{q}\right)^{\frac{1}{q}}
$$

is finite (the sum should be replaced by the supremum if $q=\infty$ ). 
The $J$-interpolation space is formed by all elements $a \in \Sigma(\bar{A})$ which can be represented as

$$
a=\sum_{(m, n) \in Z^{2}} u_{m, n} \quad(\text { convergence in } \Sigma(\bar{A}))
$$

with $\left(u_{m, n}\right) \subset \Delta(\bar{A})$ and

$$
\left(\sum_{(m, n) \in Z^{2}}\left(2^{-\alpha m-\beta n} J\left(2^{m}, 2^{n} ; u_{m, n}\right)\right)^{q}\right)^{\frac{1}{q}}<\infty .
$$

The norm in $\bar{A}_{(\alpha, \beta), q ; J}$ is

$$
\|a\|_{(\alpha, \beta), q ; J}=\inf \left\{\left(\sum_{(m, n) \in Z^{2}}\left(2^{-\alpha m-\beta n} J\left(2^{m}, 2^{n} ; u_{m, n}\right)\right)^{q}\right)^{\frac{1}{q}}\right\}
$$

where the infimum is taken over all representations $\left(u_{m, n}\right)$ as above. It is possible to give continuous characterizations for the spaces $\bar{A}_{(\alpha, \beta), q ; K}$ and $\bar{A}_{(\alpha, \beta), q ; J}$ using integrals instead of sums, but they will not be required here (see [8] for more details).

Note that the real interpolation space $\left(A_{0}, A_{1}\right)_{\theta, q}$ can be described by a similar scheme, but replacing the polygon $\Pi$ by the segment $[0,1]$, the $N$-tuple by a couple $\left(A_{0}, A_{1}\right)$ and $(\alpha, \beta)$ by a point $\theta \in(0,1)$. In the case of couples, it is well known that $J$ and $K$-spaces coincide with equivalence of norms, i.e.

$$
\left(A_{0}, A_{1}\right)_{\theta, q ; K}=\left(A_{0}, A_{1}\right)_{\theta, q ; J}=\left(A_{0}, A_{1}\right)_{\theta, q}
$$

(see [1] or [13]). However, working with $N$-tuples $(N \geq 3), K$ - and $J$-spaces do not agree in general. We only have the continuous inclusion $\bar{A}_{(\alpha, \beta), q ; J} \hookrightarrow \bar{A}_{(\alpha, \beta), q ; K}$ (see $[\mathbf{8}$, Theorem 1.3]).

Let $\bar{B}=\left\{B_{1}, \ldots, B_{n}\right\}$ be another Banach $N$-tuple, which we also think of as sitting on the vertices of another copy of $\Pi$. By $T: \bar{A} \rightarrow \bar{B}$ we mean a bounded linear operator from $\Sigma(\bar{A})$ into $\Sigma(\bar{B})$ whose restriction to each $A_{j}$ defines a bounded operator from $A_{j}$ into $B_{j}, j=1, \ldots, N$. We denote the norm of $T: A_{j} \rightarrow B_{j}$ by $\|T\|_{j}$.

It is not hard to check that if $T: \bar{A} \rightarrow \bar{B}$, then the restriction of $T$ to $\bar{A}_{(\alpha, \beta), q ; K}$ gives a bounded operator

$$
T: \bar{A}_{(\alpha, \beta), q ; K} \rightarrow \bar{B}_{(\alpha, \beta), q ; K}
$$

According to [6, Theorem 1.9], its norm can be estimated by

$$
\|T\|_{(\alpha, \beta), q ; K}=\left\|T: \bar{A}_{(\alpha, \beta), q ; K} \rightarrow \bar{B}_{(\alpha, \beta), q ; K}\right\| \leq C \max _{\{i, j, k\} \in \mathcal{P}_{(\alpha, \beta)}}\left\{\|T\|_{i}^{c_{i}}\|T\|_{j}^{c_{j}}\|T\|_{k}^{c_{k}}\right\} .
$$

Here $C$ is a constant that depends only on $(\alpha, \beta)$ and $\Pi, \mathcal{P}_{(\alpha, \beta)}$ stands for the collection of all triples $\{i, j, k\}$ such that the point $(\alpha, \beta)$ belongs to the interior of the triangle $\overline{P_{i} P_{j} P_{k}}$ and $\left(c_{i}, c_{i}, c_{k}\right)$ are the barycentric coordinates of $(\alpha, \beta)$ with respect to the 
vertices $\left\{P_{i}, P_{j}, P_{k}\right\}$. A similar estimate holds for the restriction of $T$ to the $J$-spaces. If we consider instead the operator $T$ acting from a $J$-space into a $K$-space, then it was shown in [6, Theorem 3.2] that

$$
\left\|T: \bar{A}_{(\alpha, \beta), q ; J} \rightarrow \bar{B}_{(\alpha, \beta), q ; K}\right\| \leq C \prod_{j=1}^{N}\|T\|_{j}^{\theta_{j}}
$$

where $\bar{\theta}=\left(\theta_{1}, \ldots, \theta_{N}\right)$ are some barycentric coordinates of $(\alpha, \beta)$ with respect to the vertices $P_{1}, \ldots, P_{N}$ of $\Pi$ (i.e. $0<\theta_{1}, \ldots, \theta_{N}<1, \sum_{j=1}^{N} \theta_{j}=1$ and $\sum_{j=1}^{N} \theta_{j} P_{j}=(\alpha, \beta)$ ), and $\mathrm{C}$
is a constant depending only on $\bar{\theta}$.

Inequality (1) for $J$-spaces yields

$$
\|a\|_{(\alpha, \beta), q ; J \leq C_{1}} \max _{\{i, j, k\} \in \mathcal{P}_{(\alpha, \beta)}}\left\{\|a\|_{A_{i}}^{c_{i}}\|a\|_{A_{j}}^{c_{j}}\|a\|_{A_{k}}^{c_{k}}\right\}, a \in \Delta(\bar{A}),
$$

while for the $K$-norm it follows from (2) that

$$
\|a\|_{(\alpha, \beta), q ; K} \leq C_{2} \prod_{j=1}^{N}\|a\|_{A_{j}}^{\theta_{j}}, a \in \Delta(\bar{A}) .
$$

Given any double sequence of Banach spaces $\left(W_{m, n}\right)_{(m, n) \in Z^{2}}$ and any sequence of non-negative numbers $\left(\lambda_{m, n}\right)_{(m, n) \in \mathrm{Z}^{2}}$ we write $\ell_{q}\left(\lambda_{m, n} W_{m, n}\right)$ to designate the vectorvalued $\ell_{q}$ space modelled on the $W_{m, n}$, that is to say,

$$
\begin{array}{r}
\ell_{q}\left(\lambda_{m, n} W_{m, n}\right)=\left\{w=\left(w_{m}\right): w_{m, n} \in W_{m, n}\right. \text { and } \\
\left.\|w\|_{\ell_{q}\left(\lambda_{m, n} W_{m, n}\right)}=\left(\sum_{(m, n) \in Z^{2}}\left(\lambda_{m, n}\left\|w_{m, n}\right\|_{W_{m, n}}\right)^{q}\right)^{\frac{1}{q}}<\infty\right\} .
\end{array}
$$

\section{Estimates for the measure of non-compactness.}

Theorem 2.1. Let $\Pi=\overline{P_{1} \ldots P_{N}}$ be a convex polygon with vertices $P_{j}=\left(x_{j}, y_{j}\right)$, let $(\alpha, \beta) \in$ Int $\Pi$ and $1 \leq q \leq \infty$. There exist constants $\gamma>0$ and $0<\tau<1$, depending only on $\Pi$ and $(\alpha, \beta)$, such that for any $N$-tuples, $\bar{A}=\left\{A_{1}, \ldots, A_{N}\right\}$ and $\bar{B}=\left\{B_{1}, \ldots, B_{N}\right\}$, and any operator $T: \bar{A} \rightarrow \bar{B}$ the measure of non-compactness of the interpolated operator can be estimated by

$$
\beta\left(T: \bar{A}_{(\alpha, \beta), q ; J} \rightarrow \bar{B}_{(\alpha, \beta), q ; K}\right) \leq \gamma \min _{1 \leq i \leq N}\left\{\beta\left(T: A_{i} \rightarrow B_{i}\right)\right\}^{\tau} \max _{1 \leq i \leq N}\left\{\|T\|_{i}\right\}^{1-\tau} .
$$

Proof. As we pointed out in the Introduction, we shall use in the proof some ideas developed in [7] in order to use efficiently the estimate established in [5] for the real method.

First of all, by [7, Remark 4.1], we can assume without loss of generality that $\Pi$ 
is such that $P_{1}=(0,0), P_{2}=(1,0)$ and $P_{N}=(0,1)$. We can also suppose that $\beta_{1}=\min _{1 \leq j \leq N}\left\{\beta_{i}\right\}$, where $\beta_{i}=\beta\left(T: A_{i} \rightarrow B_{i}\right)$.

Since $(\alpha, \beta) \in \operatorname{Int} \Pi$, there exists $0<\theta<1$ with $\left(\alpha^{\prime}, \beta^{\prime}\right)=(\alpha / \theta, \beta / \theta) \in \operatorname{Int} \Pi$. Write $A_{i}^{\theta}=\left(A_{1}, A_{i}\right)_{\theta, 1}, B_{i}^{\theta}=\left(B_{1}, B_{i}\right)_{\theta, 1}$ and consider the $N$-tuples $\bar{A} \theta=\left\{A_{1}^{\theta}, \ldots, A_{N}^{\theta}\right\}$, $\bar{B}^{\theta}=\left\{B_{1}^{\theta}, \ldots, B_{N}^{\theta}\right\}$. According to the formula we established in [5, Theorem 1.2], the measure of non-compactness $\tilde{\beta}_{i}$ of $T: A_{i}^{\theta} \rightarrow B_{i}^{\theta}$ can be estimated by

$$
\tilde{\beta}_{i}=\beta\left(T: A_{i}^{\theta} \rightarrow B_{i}^{\theta}\right) \leq C_{\theta} \beta_{1}^{1-\theta} \beta_{i}^{\theta} .
$$

On the other hand, by [7, Theorem 4.7], we can compare spaces generated by $\bar{A}, \bar{B}$ and $(\alpha, \beta)$ with those defined by $\bar{A}^{\theta}, \bar{B}^{\theta}$ and $\left(\alpha^{\prime}, \beta^{\prime}\right)$. Namely, the following continuous inclusions hold:

$$
\bar{A}_{(\alpha, \beta), q ; J^{\hookrightarrow}} \bar{A}_{\left(\alpha^{\prime}, \beta^{\prime}\right), q ; J}^{\theta}, \quad \bar{A}_{\left(\alpha^{\prime}, \beta^{\prime}\right), q ; K}^{\theta} \bar{A}_{(\alpha, \beta), q ; K} .
$$

Hence, there is a constant $C$ depending only on $(\alpha, \beta)$ and $\Pi$ such that

$$
\beta\left(T: \bar{A}_{(\alpha, \beta), q ; J} \rightarrow \bar{B}_{(\alpha, \beta), q ; K}\right) \leq C \beta\left(T: \bar{A}_{\left(\alpha^{\prime}, \beta^{\prime}\right), q ; J}^{\theta} \rightarrow \bar{B}_{\left(\alpha^{\prime}, \beta\right), q ; K}^{\theta}\right)
$$

This shows that in order to establish the theorem it suffices to work with $\beta\left(T: \bar{A}_{\left(\alpha^{\prime}, \beta^{\prime}\right), q ; J}^{\theta} \rightarrow \bar{B}_{\left(\alpha^{\prime}, \beta^{\prime}\right), q ; K}^{\theta}\right)$. With this aim, we put

$$
G_{m, n}^{\theta}=\left(\Delta\left(\bar{A}^{\theta}\right), J\left(2^{m}, 2^{n}, . \bar{A}^{\theta}\right)\right), \quad F_{m, n}^{\theta}=\left(\sum\left(\bar{A}^{\theta}\right), K\left(2^{m}, 2^{n}, . ; \bar{A}^{\theta}\right)\right),(m, n) \in Z^{2}
$$

and we shall work with vector-valued sequence spaces modelled on these Banach spaces.

Let $\pi$ be the operator defined by $\pi\left(u_{m, n}\right)=\sum_{(m, n) \in Z^{2}} u_{m, n}$. Clearly $\pi: \ell_{1}\left(2^{-m x_{i}-n y_{i}} G_{m, n}^{\theta}\right) \rightarrow A_{i}^{\theta}$ is bounded with norm $\leq 1$ for $i=1, \ldots, N$. Moreover $\pi: \ell_{q}\left(2^{-\alpha^{\prime} m-\beta^{\prime} n} G_{m, n}^{\theta}\right) \rightarrow \bar{A}_{\left(\alpha^{\prime}, \beta^{\prime}\right), q ; J}^{\theta}$ is a metric surjection.

Consider next the operator $j$ that associates to each $b \in \Sigma\left(\bar{B}^{\theta}\right)$ the constant sequence $j(b)=(\ldots, b, b, b, \ldots)$. This time $j: B_{i}^{\theta} \rightarrow \ell_{\infty}\left(2^{-m x_{i}-n y_{i}} F_{m, n}^{\theta}\right)$ is bounded with norm $\leq 1$ for $i=1, \ldots, N$, and $j: \bar{B}_{\left(\alpha^{\prime}, \beta^{\prime}\right), q ; K}^{\theta} \rightarrow \ell_{q}\left(2^{-\alpha^{\prime} m-\beta^{\prime} n} F_{m, n}\right)$ is a metric injection.

So we have the following diagram of bounded operators.

$$
\begin{aligned}
& \ell_{1}\left(2^{-m x_{1}-n y_{1}} G_{m, n}^{\theta}\right) \stackrel{\pi}{\rightarrow} A_{1}^{\theta} \stackrel{T}{\rightarrow} B_{1}^{\theta} \stackrel{j}{\rightarrow} \ell_{\infty}\left(2^{-m x_{1}-n y_{1}} F_{m, n}^{\theta}\right) \\
& \frac{\ell_{1}\left(2^{-m x_{N}-n y_{N}} G_{m, n}^{\theta}\right) \stackrel{\pi}{\rightarrow} A_{N}^{\theta} \stackrel{T}{\rightarrow} B_{N}^{\theta} \stackrel{j}{\rightarrow} \ell_{\infty}\left(2^{-m x_{N}-n y_{N}} F_{m, n}^{\theta}\right)}{\ell_{q}\left(2^{-\alpha^{\prime} m-\beta^{\prime} n} G_{m, n}^{\theta}\right) \stackrel{\pi}{\rightarrow} \bar{A}_{\left(\alpha^{\prime}, \beta^{\prime}\right), q ; J}^{\theta} \stackrel{T}{\rightarrow} \bar{B}_{\left(\alpha^{\prime}, \beta^{\prime}\right), q ; K}^{\theta} \stackrel{j}{\rightarrow} \ell_{q}\left(2^{-\alpha^{\prime} m-\beta^{\prime} n} F_{m, n}^{\theta}\right)}
\end{aligned}
$$

Write $\hat{\ell}_{1}=\left\{\ell_{1}\left(2^{-m x_{1}-n y_{1}} G_{m, n}^{\theta}\right), \ldots, \ell_{1}\left(2^{-m x_{N}-n y_{N}} G_{m, n}^{\theta}\right)\right\}, \hat{\ell}_{\infty}=\left\{\ell_{\infty}\left(2^{-m x_{1}-n y_{1}} G_{m, n}^{\theta}\right), \ldots\right.$, $\left.\ell_{\infty}\left(2^{-m x_{N}-n y_{N}} G_{m, n}^{\theta}\right)\right\}$ and put $\hat{T}=j T \pi$. Using the properties mentioned above of $j$ and 
$\pi$, we get

$$
\beta\left(T: \bar{A}_{\left(\alpha^{\prime}, \beta^{\prime}\right), q ; J}^{\theta} \rightarrow \bar{B}_{\left(\alpha^{\prime}, \beta^{\prime}\right), q ; K}^{\theta}\right) \leq 2 \beta\left(\hat{T}: \ell_{q}\left(2^{-\alpha^{\prime} m-\beta^{\prime} n} G_{m, n}^{\theta}\right) \rightarrow \ell_{q}\left(2^{-\alpha^{\prime} m-\beta^{\prime} n} F_{m, n}^{\theta}\right)\right) .
$$

We write, for simplicity, $\beta(\hat{T})=\beta\left(\hat{T}: \ell_{q}\left(2^{-\alpha^{\prime} m-\beta^{\prime} n} G_{m, n}^{\theta}\right) \rightarrow \ell_{q}\left(2^{-\alpha^{\prime} m-\beta^{\prime} n} F_{m, n}^{\theta}\right)\right)$. In order to estimate this value let us introduce on $\hat{\ell}_{1}$ families of operators $\left\{P_{k}^{(r)}\right\}_{k=1}^{\infty}$, $r=0,1,2,3,4$ defined by $P_{k}^{(r)}\left(\left(\xi_{m, n}\right)\right)=\left(u_{m, n}\right)$ where

$$
u_{m, n}=\left\{\begin{array}{cc}
\xi_{m, n} & \text { if }(m, n) \in \Omega_{k}^{(r)} \\
0 & \text { otherwise }
\end{array}\right.
$$

and where the sets $\left\{\Omega_{k}{ }^{(r)}\right\}$ are given by

$$
\begin{aligned}
& \Omega_{k}^{(0)}=\left\{(m, n) \in Z^{2}:|m|<k,|n|<k\right\}, \\
& \Omega_{k}^{(1)}=\left\{(m, n) \in Z^{2}: m \leq-k,|n|<k\right\}, \\
& \Omega_{k}^{(2)}=\left\{(m, n) \in Z^{2}: m \geq k,|n|<k\right\}, \\
& \Omega_{k}^{(3)}=\left\{(m, n) \in Z^{2}: n \leq-k\right\}, \\
& \Omega_{k}^{(4)}=\left\{(m, n) \in Z^{2}: n \geq k\right\} .
\end{aligned}
$$

It is not hard to check that the following properties hold.

(I) The identity operator on $\Sigma\left(\hat{\ell}_{1}\right)$ can be decomposed as

$$
I=\sum_{r=0}^{4} P_{k}^{(r)}, \quad k=1,2, \ldots
$$

(II) They are uniformly bounded, i.e.

$$
\left\|P_{k}^{(r)}: \ell_{1}\left(2^{-m x_{i}-n y_{i}} G_{m, n}^{\theta}\right) \rightarrow \ell_{1}\left(2^{-m x_{i}-n y_{i}} G_{m, n}^{\theta}\right)\right\|=1
$$

for any $k \in \mathrm{N}, 0 \leq r \leq 4,1 \leq i \leq N$.

(III) For each $k \in \mathrm{N}$, we have that

$$
\begin{gathered}
P_{k}^{(1)}: \ell_{1}\left(2^{-m} G_{m, n}^{\theta}\right) \rightarrow \ell_{1}\left(G_{m, n}^{\theta}\right), \\
P_{k}^{(2)}: \ell_{1}\left(G_{m, n}^{\theta}\right) \rightarrow \ell_{1}\left(2^{-m} G_{m, n}^{\theta}\right), \\
P_{k}^{(3)}: \ell_{1}\left(2^{-n} G_{m, n}^{\theta}\right) \rightarrow \ell_{1}\left(G_{m, n}^{\theta}\right), \\
P_{k}^{(4)}: \ell_{1}\left(G_{m, n}^{\theta}\right) \rightarrow \ell_{1}\left(2^{-n} G_{m, n}^{\theta}\right),
\end{gathered}
$$

and their norms are equal to $2^{-k}$

(IV) For each $k \in \mathrm{N}, P_{k}^{(0)}: \Sigma\left(\hat{\ell}_{1}\right) \rightarrow \Delta\left(\hat{\ell}_{1}\right)$ is bounded. 
Since $\hat{T}=\hat{T} P_{k}^{(0)}+\hat{T} P_{k}^{(1)}+\hat{T} P_{k}^{(2)}+\hat{T} P_{k}^{(3)}+\hat{T} P_{k}^{(4)}$, we get

$$
\beta(\hat{T}) \leq \beta\left(\hat{T} P_{k}^{(0)}\right)+\sum_{r=1}^{4}\left\|\hat{T} P_{k}^{(r)}\right\|
$$

where all the operators are considered from $\ell_{q}\left(2^{-\alpha^{\prime} m-\beta^{\prime} n} G_{m, n}^{\theta}\right)$ into $\ell_{q}\left(2^{-\alpha^{\prime} m-\beta^{\prime} n} F_{m, n}^{\theta}\right)$. Let us estimate each of these terms. We start with $\beta\left(\hat{T} P_{k}^{0}\right)$.

Let $\ell_{q}^{(2 k-1)^{2}}$ be $R^{(2 k-1)^{2}}$ with the $\ell_{q}$-norm. Since $\ell_{q}^{(2 k-1)^{2}}$ is finite dimensional, given any $\varepsilon>0$, there exists a finite set $\left\{\mu^{r}\right\}_{r=1}^{l} \subseteq \mathcal{U}_{\ell_{q}^{(2 k-1)^{2}}}$ such that for any $\lambda \in \mathcal{U}_{\ell_{q}^{(2 k-1)^{2}}}$

$$
\min _{1 \leq r \leq l}\left\{\left\|\lambda-\mu^{r}\right\|_{\ell_{q}^{(2 k-1)^{2}}}\right\} \leq \varepsilon .
$$

Given any $u=\left(u_{m, n}\right) \in \mathcal{U}_{\ell_{q}\left(2^{-\alpha^{\prime} m-\beta^{\prime} n} G_{m, n}^{\theta}\right)}$, since

$$
\left\|\left(2^{-\alpha^{\prime} m-\beta^{\prime} n} J\left(2^{m}, 2^{n} ; u_{m, n}\right)\right)_{|m|,|n|<k}\right\|_{\ell_{q}^{(2 k-1)^{2}}} \leq\left(\sum_{(m, n) \in Z^{2}}\left(2^{-\alpha^{\prime} m-\beta^{\prime} n} J\left(2^{m}, 2^{n} ; u_{m, n}\right)\right)^{q}\right)^{1 / q} \leq 1
$$

we can find $r \in[1, l]$ satisfying that

$$
2^{-\alpha^{\prime} m-\beta^{\prime} n} J\left(2^{m}, 2^{n} ; u_{m, n}\right) \leq \mu_{m, n}^{r}+\varepsilon
$$

for any $m, n$ with $|m|,|n|<k$, where $\mu^{r}=\left(\mu_{m, n}^{r}\right)_{|m|,|n|<k}$. Hence

$$
\left\|u_{m, n}\right\|_{A_{i}^{\theta}} \leq\left(\mu_{m, n}^{r}+\varepsilon\right) 2^{\left(\alpha^{\prime}-x_{i}\right) m+\left(\beta^{\prime}-y_{i}\right) n}, \quad 1 \leq i \leq N,|m|,|n|<k .
$$

According to the definition of $\tilde{\beta}_{i}$, if $\tilde{k}_{i}>\tilde{\beta}_{i}$, we can find a finite set of vectors $\left\{b^{i, v}\right\} \subseteq B_{i}^{\theta}, v=1, \ldots, h_{i}, 1 \leq i \leq N$, such that

$$
\begin{array}{r}
\min _{1 \leq v \leq h_{i}}\left\{\left\|T\left(u_{m, n}\right)-\left(\mu_{m, n}^{r}+\varepsilon\right) 2^{\left(\alpha^{\prime}-x_{i}\right) m+\left(\beta^{\prime}-y_{i}\right) n} b^{i, v}\right\|_{B_{i}^{\theta}}\right\} \\
\leq \tilde{k}_{i}\left(\mu_{m, n}^{r}+\varepsilon\right) 2^{\left(\alpha^{\prime}-x_{i}\right) m+\left(\beta^{\prime}-y_{i}\right) n}, 1 \leq i \leq N .
\end{array}
$$

So, for each $|m|,|n|<k$, there is a finite set $\left\{d_{m, n}^{p}\right\} \subseteq B_{1}^{\theta} \cap \cdots \cap B_{N}^{\theta}$ of, say, $w=w(m, n)$ vectors such that for some $p$

$$
\left\|T\left(u_{m, n}\right)-d_{m, n}^{p}\right\|_{B_{i}^{\theta}} \leq 2 \tilde{k}_{i}\left(\mu_{m, n}^{r}+\varepsilon\right) 2^{\left(\alpha^{\prime}-x_{i}\right) m+\left(\beta^{\prime}-y_{i}\right) n}, 1 \leq i \leq N .
$$

Let

$$
\mathcal{D}=\left\{\sum_{|m|,|n|<k} d_{m, n}^{p}: p=p(m, n) \in[1, w(m, n)]\right\} .
$$

Then $\mathcal{D}$ is a finite subset of $\bar{B}_{\left(\alpha^{\prime}, \beta^{\prime}\right), q ; K}^{\theta}$ and is such that for each $u=\sum_{(m, n) \in Z^{2}} u_{m, n} \in \mathcal{U}_{\ell_{q}\left(2^{-\alpha^{\prime} m-\beta^{\prime} n} G_{m, n}^{\theta}\right)}$ there exists some $\sum_{|m|,|n|<k} d_{m, n}^{p} \in \mathcal{D}$ with 


$$
\begin{aligned}
& K\left(2^{s}, 2^{t} ; \sum_{|m|,|n|<k}\left(T\left(u_{m, n}\right)-d_{m, n}^{p}\right)\right) \\
& \leq \sum_{|m|,|n|<k} K\left(2^{s}, 2^{t} ; T\left(u_{m, n}\right)-d_{m, n}^{p}\right) \\
& \leq \sum_{|m|,|n|<k} \min _{1 \leq i \leq N}\left\{2^{s x_{i}} 2^{t y_{i}}\left\|T\left(u_{m, n}\right)-d_{m, n}^{p}\right\|_{\left.B_{i}^{\theta}\right\}}\right. \\
& \leq \sum_{|m|,|n|<k} 2 \min _{1 \leq i \leq N}\left\{2^{s x_{i}} 2^{t y_{i}} \tilde{k}_{i}\left(\mu_{m, n}^{r}+\varepsilon\right) 2^{\left(\alpha^{\prime}-x_{i}\right) m+\left(\beta^{\prime}-y_{i}\right) n}\right\} \\
& =\sum_{(m, n) \in Z^{2}} 2\left(\tilde{\mu}_{m, n}^{r}+\varepsilon\right) \min _{1 \leq i \leq N}\left\{2^{(s-m) x_{i}+(t-n) y_{i}+\alpha^{\prime} m+\beta^{\prime} n} \tilde{k_{i}}\right\} \\
& =\sum_{\left(m^{\prime}, n^{\prime}\right) \in Z^{2}} 2\left(\tilde{\mu}_{s-m^{\prime}, t-n^{\prime}}^{r}+\varepsilon\right) \min _{1 \leq i \leq N}\left\{2^{m^{\prime} x_{i}+n^{\prime} y_{i}+\alpha^{\prime}\left(s-m^{\prime}\right)+\beta^{\prime}\left(t-n^{\prime}\right)} \tilde{k}_{i}\right\}
\end{aligned}
$$

where

$$
\mu_{m, n}^{r}=\left\{\begin{array}{cc}
\mu_{m, n}^{r} & \text { if }|m|,|n|<k \\
-\varepsilon & \text { otherwise }
\end{array}\right.
$$

Thus

$$
\begin{aligned}
& \left\|T \pi P_{k}^{(0)}(u)-\sum_{|m|,|n|<k} d_{m, n}^{p}\right\|_{\left(\alpha^{\prime}, \beta^{\prime}\right), q ; K} \\
& =\left[\sum_{(s, t) \in Z^{2}}\left(2^{-\alpha^{\prime} s-\beta^{\prime} t} K\left(2^{s}, 2^{t} ; \sum_{|m|,|n|<k}\left(T\left(u_{m, n}\right)-d_{m, n}^{p}\right)\right)\right)^{q}\right]^{1 / q} \\
& \leq\left[\sum_{(s, t) \in Z^{2}}\left(2^{-\alpha^{\prime} s-\beta^{\prime} t} \sum_{\left(m^{\prime}, n^{\prime}\right) \in Z^{2}} 2\left(\tilde{\mu}_{s-m^{\prime}, t-n^{\prime}}^{r}+\varepsilon\right) \min _{1 \leq i \leq N}\left\{2^{m^{\prime} x_{i}+n^{\prime} y_{i}+\alpha^{\prime}\left(s-m^{\prime}\right)+\beta^{\prime}\left(t-n^{\prime}\right)} \tilde{k}_{i}\right\}\right)^{q}\right]^{1 / q} \\
& =\left[\sum_{(s, t) \in Z^{2}}\left(\sum_{\left(m^{\prime}, n^{\prime}\right) \in Z^{2}} 2\left(\tilde{\mu}_{s-m^{\prime}, t-n^{\prime}}^{r}+\varepsilon\right) \min _{1 \leq i \leq N}\left\{2^{m^{\prime} x_{i}+n^{\prime} y_{i}-\alpha^{\prime} m^{\prime}-\beta^{\prime} n^{\prime}} \tilde{k}_{i}\right\}\right)^{q}\right]^{1 / q} \\
& \leq 2 \sum_{\left(m^{\prime}, n^{\prime}\right) \in Z^{2}}\left(\sum_{(s, t) \in Z^{2}}\left(\tilde{\mu}_{s-m^{\prime}, t-n^{\prime}}^{r}+\varepsilon\right)^{q} \min _{1 \leq i \leq N}\left\{2^{m^{\prime} x_{i}+n^{\prime} y_{i}-\alpha^{\prime} m^{\prime}-\beta^{\prime} n^{\prime}} \tilde{k}_{i}\right\}^{q}\right)^{1 / q} \\
& =2 \sum_{\left(m^{\prime}, n^{\prime}\right) \in Z^{2}}\left[\min _{1 \leq i \leq N}\left\{2^{m^{\prime} x_{i}+n^{\prime} y_{i}-\alpha^{\prime} m^{\prime}-\beta^{\prime} n^{\prime}} \tilde{k}_{i}\right\}\left(\sum_{\substack{-k+m^{\prime}<s<k+m^{\prime} \\
-k+n^{\prime}<<k+n^{\prime}}}\left(\tilde{\mu}_{s-m^{\prime}, t-n^{\prime}}^{r}+\varepsilon\right)^{q}\right)^{1 / q}\right] \\
& \leq 2\left(1+\varepsilon(2 k-1)^{2 / q}\right) \sum_{\left(m^{\prime}, n^{\prime}\right) \in Z^{2}} 2^{-\alpha^{\prime} m^{\prime}-\beta^{\prime} n^{\prime}} \min _{1 \leq i \leq N}\left\{2^{m^{\prime} x_{i}+n^{\prime} y_{i}} \tilde{k}_{i}\right\}
\end{aligned}
$$

To evaluate the last series observe that since $\left(\alpha^{\prime}, \beta^{\prime}\right) \in \operatorname{Int} \Pi$, we can choose $\varepsilon_{1}>0$ such that $\left(\alpha^{\prime}, \beta^{\prime}\right)+\varepsilon_{1} h \in \operatorname{Int} \Pi$ for all possible vectors $h=( \pm 1, \pm 1)$. By [7, Lemma 4.2], there exist positive real numbers $\left\{\alpha_{i}(h)\right\}_{i=1}^{N}$ such that 


$$
\sum_{i=1}^{N} \alpha_{i}(h)=1 \text { and }\left(\alpha^{\prime}, \beta^{\prime}\right)+\varepsilon_{1} h=\sum_{i=1}^{N} \alpha_{i}(h) P_{i} .
$$

Taking into account that $\min _{1 \leq i \leq N} \delta_{i} \leq \prod_{i=1}^{N} \delta_{i}^{v_{i}}$ for $\delta_{i}, v_{i}>0$ with $\sum_{i=1}^{N} v_{i}=1$, we obtain

$$
2^{-\alpha^{\prime} m^{\prime}-\beta^{\prime} n^{\prime}} \min _{1 \leq i \leq N}\left\{2^{m^{\prime} x_{i}+n^{\prime} y_{i}} \tilde{k}_{i}\right\} \leq 2^{-\alpha^{\prime} m^{\prime}-\beta^{\prime} n^{\prime}} \prod_{i=1}^{N}\left(2^{m^{\prime} x_{i}+n^{\prime} y_{i}} \tilde{k_{i}}\right)^{\alpha_{i}(h)}=2^{\varepsilon_{1}<(m, n), h>} \prod_{i=1}^{N} \tilde{k}_{i}^{\alpha_{i}(h)}
$$

where $<,>$ stands for the inner product of $\mathrm{R}^{2}$.

Put $\tau_{1}=\min \left\{\alpha_{i}(h): 1 \leq i \leq N, h=( \pm 1, \pm 1)\right\}$. Then we have

$$
\begin{aligned}
\prod_{i=1}^{N} \tilde{k}_{i}^{\alpha_{i}(h)} & =\max _{1 \leq i \leq N}\left\{\tilde{k}_{i}\right\} \prod_{i=1}^{N}\left(\frac{\tilde{k}_{i}}{\max _{1 \leq i \leq N}\left\{\tilde{k}_{i}\right\}}\right)^{\alpha_{i}(h)} \\
& \leq \max _{1 \leq i \leq N}\left\{\tilde{k}_{i}\right\}\left(\frac{\min _{1 \leq i \leq N}\left\{\tilde{k}_{i}\right\}}{\max _{1 \leq i \leq N}\left\{\tilde{k}_{i}\right\}}\right)^{\tau_{1}} \\
& =\left(\min _{1 \leq i \leq N}\left\{\tilde{k}_{i}\right\}\right)^{\tau_{1}}\left(\max _{1 \leq i \leq N}\left\{\tilde{k}_{i}\right\}\right)^{1-\tau_{1}} .
\end{aligned}
$$

Taking the minimum over all $h=( \pm 1, \pm 1)$ we obtain

$$
2^{-\alpha^{\prime} m^{\prime}-\beta^{\prime} n^{\prime}} \min _{1 \leq i \leq N}\left\{2^{m^{\prime} x_{i}+n^{\prime} y_{i}} \tilde{k}_{i}\right\} \leq 2^{-\left|m^{\prime}\right| \varepsilon_{1}-\left|n^{\prime}\right| \varepsilon_{1}}\left(\min _{1 \leq i \leq N}\left\{\tilde{k}_{i}\right\}\right)^{\tau_{1}}\left(\max _{1 \leq i \leq N}\left\{\tilde{k}_{i}\right\}\right)^{1-\tau_{1}} .
$$

This implies that

$$
\sum_{\left(m^{\prime}, n^{\prime}\right) \in Z^{2}} 2^{-\alpha^{\prime} m^{\prime}-\beta^{\prime} n^{\prime}} \min _{1 \leq i \leq N}\left\{2^{m^{\prime} x_{i}+n^{\prime} y_{i}} \tilde{k}_{i}\right\} \leq\left(\min _{1 \leq i \leq N}\left\{\tilde{k}_{i}\right\}\right)^{\tau_{1}}\left(\max _{1 \leq i \leq N}\left\{\tilde{k}_{i}\right\}\right)^{1-\tau_{1}} \sum_{\left(m^{\prime}, n^{\prime}\right) \in Z^{2}} 2^{-\left|m^{\prime}\right| \varepsilon_{1}-\left|n^{\prime}\right| \varepsilon_{1}},
$$

and therefore,

$$
\beta\left(\hat{T} P_{k}^{(0)}\right) \leq \beta\left(T \pi_{k}^{(0)}\right) \leq 2\left(\sum_{\left(m^{\prime}, n^{\prime}\right) \in Z^{2}} 2^{-\left|m^{\prime}\right| \varepsilon_{1}-\left|n^{\prime}\right| \varepsilon_{1}}\right)\left(\min _{1 \leq i \leq N}\left\{\tilde{\beta}_{i}\right\}\right)^{\tau_{1}}\left(\max _{1 \leq i \leq N}\left\{\tilde{\beta}_{i}\right\}\right)^{1-\tau_{1}} .
$$

Put $\gamma_{1}=2\left(\sum_{\left(m^{\prime}, n^{\prime}\right) \in Z^{2}} 2^{-\left|m^{\prime}\right| \varepsilon_{1}-\left|n^{\prime}\right| \varepsilon_{1}}\right)$. Recalling that $\tilde{\beta}_{i} \leq C_{\theta} \beta_{1}^{1-\theta} \beta_{i}^{\theta}$ with $\beta_{1}=\min _{1 \leq i \leq N}\left\{\beta_{i}\right\}$, we conclude

$$
\beta\left(\hat{T} P_{k}^{(0)}\right) \leq \gamma_{1} C_{\theta} \beta_{1}^{\tau_{1}} \beta_{1}^{(1-\theta)\left(1-\tau_{1}\right)}\left(\max _{1 \leq i \leq N}\left\{\beta_{i}\right\}\right)^{\theta\left(1-\tau_{1}\right)}=\gamma_{1} C_{\theta} \beta_{1}^{1-\theta+\theta \tau_{1}}\left(\max _{1 \leq i \leq N}\left\{\beta_{i}\right\}\right)^{\theta\left(1-\tau_{1}\right)}
$$

Next we estimate the norm of the operator 


$$
\hat{T} P_{k}^{(1)}: \ell_{q}\left(2^{-\alpha^{\prime} m-\beta^{\prime} n} G_{m, n}^{\theta}\right) \rightarrow \ell_{q}\left(2^{-\alpha^{\prime} m-\beta^{\prime} n} F_{m, n}^{\theta}\right) .
$$

The arguments given in [8, Theorem 3.1] show that

$$
\begin{aligned}
& \ell_{q}\left(2^{-\alpha^{\prime} m-\beta^{\prime} n} G_{m, n}^{\theta}\right) \rightarrow\left(\hat{\ell}_{1}\right)_{\left(\alpha^{\prime}, \beta^{\prime}\right), q ; J} \\
& \left(\hat{\ell}_{\infty}\right)_{\left(\alpha^{\prime}, \beta^{\prime}\right), q ; K} \rightarrow \ell_{q}\left(2^{-\alpha^{\prime} m-\beta^{\prime} n} F_{m, n}^{\theta}\right)
\end{aligned}
$$

with norms $\leq 1$. If $\bar{\theta}=\left(\theta_{1}, \ldots, \theta_{N}\right)$ are some barycentric coordinates of $\left(\alpha^{\prime}, \beta^{\prime}\right)$ with respect to $P_{1}, \ldots, P_{N}$, it follows from (2) and (I) that

$$
\begin{aligned}
\left\|\hat{T} P_{k}^{(1)}\right\| \leq\left\|\hat{T} P_{k}^{(1)}\right\|_{\left(\hat{\ell}_{1}\right)_{\left(\alpha^{\prime}, \beta^{\prime}\right), q, J},\left(\hat{\ell}_{\infty}\right)_{\left(\alpha^{\prime}, \beta^{\prime}\right), q, K}} & \leq C\left\|\hat{T} P_{k}^{(1)}\right\|_{2}^{\theta_{2}} \max _{1 \leq i \leq N}\left\{\left\|\hat{T} P_{k}^{(1)}\right\|_{i}\right\}^{1-\theta_{2}} \\
& \leq C\left\|\hat{T} P_{k}^{(1)}\right\|_{2}^{\theta_{2}} \max _{1 \leq i \leq N}\left\{\|T\|_{i}\right\}^{1-\theta_{2}} .
\end{aligned}
$$

Further since

$$
\left\|\hat{T} P_{1}^{(1)}\right\|_{2} \geqslant\left\|\hat{T} P_{2}^{(1)}\right\|_{2} \geqslant \cdots \geqslant 0
$$

there exists $\lambda \geq 0$ such that $\left\|\hat{T} P_{k}^{(1)}\right\|_{2} \rightarrow \lambda$ as $k \rightarrow \infty$. Choose vectors $\left(u^{k}\right)_{k \in N} \subset \mathcal{U}_{\ell_{1}\left(2^{-m} G_{m, n}^{\theta}\right)}$ such that

$$
\left\|\hat{T} P_{k}^{(1)}\left(u^{k}\right)\right\|_{\ell_{\infty}\left(2^{-m} F_{m, n}^{\theta}\right)} \rightarrow \lambda \text { as } k \rightarrow \infty
$$

By the definition of $\tilde{\beta}_{2}$, given any $\varepsilon>0$, there exists a finite set $\left\{b_{1}{ }^{2}, b_{2}{ }^{2}, \ldots, b_{s}{ }^{2}\right\}$ in $B_{2}^{\theta}$ such that

$$
T \pi\left(\mathcal{U}_{\ell_{1}\left(2^{-m} G_{m, n}^{\theta}\right.}\right) \subseteq \bigcup_{r=1}^{s}\left\{b_{r}^{2}+\left(\tilde{\beta}_{2}+\varepsilon\right) \mathcal{U}_{B_{2}^{\theta}}\right\}
$$

For some subsequence $\left(k^{\prime}\right) \subset \mathrm{N}$ and some $r$, say $r=1$, it follows that

$$
T \pi P_{k^{\prime}}^{(1)}\left(u^{k^{\prime}}\right) \in\left\{b_{1}^{2}+\left(\tilde{\beta}_{2}+\varepsilon\right) \mathcal{U}_{B_{2}^{\theta}}\right\} \text { for all } k^{\prime} .
$$

Using property (III), we have that for any $m, n \in \mathrm{Z}$

$$
\begin{aligned}
2^{-m} K\left(2^{m}, 2^{n} ; b_{1}^{2}\right) & \leq 2^{-m}\left(2^{m}\left\|b_{1}^{2}-T \pi P_{k^{\prime}}^{(1)}\left(u^{k^{\prime}}\right)\right\|_{B_{2}^{\theta}}+\left\|T \pi P_{k^{\prime}}^{(1)}\left(u^{k^{\prime}}\right)\right\|_{B_{1}^{\theta}}\right) \\
& \leq\left(\tilde{\beta}_{2}+\varepsilon\right)+2^{-m-k^{\prime}}\|T\|_{1} \rightarrow \tilde{\beta}_{2}+\varepsilon \text { as } k^{\prime} \rightarrow \infty .
\end{aligned}
$$

This implies

$$
\left\|j\left(b_{1}^{2}\right)\right\|_{\ell_{\infty}\left(2^{-m} F_{m, n}^{\theta}\right)}=\sup _{(m, n) \in Z^{2}}\left\{2^{-m} K\left(2^{m}, 2^{n} ; b_{1}^{2}\right)\right\} \leq \tilde{\beta}_{2}+\varepsilon,
$$

whence 


$$
\begin{aligned}
\lambda & =\lim _{k^{\prime} \rightarrow \infty}\left\|\hat{T} P_{k^{\prime}}^{(1)}\left(u^{k^{\prime}}\right)\right\|_{\ell_{\infty}\left(2^{-m} F_{m, n}^{\theta}\right)} \\
& \leq \sup _{k^{\prime}}\left[\left\|\hat{T} P_{k^{\prime}}^{(1)}\left(u^{k^{\prime}}\right)-j\left(b_{1}^{2}\right)\right\|_{\ell_{\infty}\left(2^{-m} F_{m, n}^{\theta}\right)}+\left\|j\left(b_{1}^{2}\right)\right\|_{\ell_{\infty}\left(2^{-m} F_{m, n}^{\theta}\right)}\right] \leq 2\left(\tilde{\beta}_{2}+\varepsilon\right) .
\end{aligned}
$$

Given any $\varepsilon>0$, there then exists $k_{1} \in \mathrm{N}$ such that for all $k \geq k_{1}$,

$$
\left\|\hat{T} P_{k}^{(1)}\right\|_{2}^{\theta_{2}} \leq\left(2 \tilde{\beta}_{2}\right)^{\theta_{2}}+\varepsilon
$$

and so

$$
\left\|\hat{T} P_{k}^{(1)}\right\| \leq C\left(2 \tilde{\beta}_{2}\right)^{\theta_{2}} \max _{1 \leq i \leq N}\left\{\|T \mid\|_{i}\right\}^{1-\theta_{2}}+\varepsilon \leq C 2^{\theta_{2}} \beta_{1}^{(1-\theta) \theta_{2}} \beta_{2}^{\theta \theta_{2}} \max _{1 \leq i \leq N}\left\{\|T \mid\|_{i}\right\}^{1-\theta_{2}}+\varepsilon .
$$

Similar arguments show that

$$
\begin{aligned}
& \left\|\hat{T} P_{k}^{(2)}\right\| \leq C 2^{\theta_{1}} \beta_{1}^{\theta_{1}} \max _{1 \leq i \leq N}\left\{\|T\|_{i}\right\}^{1-\theta_{1}}+\varepsilon, \\
& \left\|\hat{T} P_{k}^{(3)}\right\| \leq C 2^{\theta_{N}} \beta_{1}^{(1-\theta) \theta_{N}} \beta_{N}^{\theta \theta_{N}} \max _{1 \leq i \leq N}\left\{\|T\|_{i}\right\}^{1-\theta_{N}}+\varepsilon \\
& \left\|\hat{T} P_{k}^{(4)}\right\| \leq C 2^{\theta_{1}} \beta_{1}^{\theta_{1}} \max _{1 \leq i \leq N}\left\{\|T\|_{i}\right\}^{1-\theta_{1}}+\varepsilon .
\end{aligned}
$$

Therefore

$$
\begin{aligned}
\beta(\hat{T}) & \leq C_{\theta} \gamma_{1} \beta_{1}^{1-\theta+\theta \tau_{1}} \max _{1 \leq i \leq N}\left\{\beta_{i}\right\}^{\theta\left(1-\tau_{1}\right)}+C 2^{\theta_{2}} \beta_{1}^{(1-\theta) \theta_{2}} \beta_{2}^{\theta \theta_{2}} \max _{1 \leq i \leq N}\left\{\|T\|_{i}\right\}^{1-\theta_{2}} \\
& +2 C 2^{\theta_{1}} \beta_{1}^{\theta_{1}} \max _{1 \leq i \leq N}\left\{\|T\|_{i}\right\}^{1-\theta_{1}}+C 2^{\theta_{N}} \beta_{1}^{(1-\theta) \theta_{N}} \beta_{N}^{\theta \theta_{N}} \max _{1 \leq i \leq N}\left\{\|T\|_{i}\right\}^{1-\theta_{N}}+4 \varepsilon .
\end{aligned}
$$

Writing $\gamma_{2}=\gamma_{1} C_{\theta}+C 2^{\theta_{2}}+C 2^{\theta_{1}+1}+C 2^{\theta_{N}}$ and $\tau=\min \left\{1-\theta+\theta \tau_{1},(1-\theta) \theta_{2}, \theta_{1}\right.$, $\left.(1-\theta) \theta_{N}\right\}$, we get

$$
\beta(\hat{T}) \leq \gamma_{2}\left(\min \left\{\beta_{i}\right\}\right)^{\tau}\left(\max \left\{\|T\|_{i}\right\}\right)^{1-\tau} .
$$

Combining this inequality with (5) and (6) we finally obtain the desired estimate

$$
\beta\left(T: \bar{A}_{(\alpha, \beta), q ; J)} \rightarrow \bar{B}_{(\alpha, \beta), q ; K}\right) \leq \gamma\left(\min \left\{\beta_{i}\right\}\right)^{\tau}\left(\max \left\{\|T\|_{i}\right\}\right)^{1-\tau} .
$$

If one of the restrictions $T: A_{i} \rightarrow B_{i}$ is compact, so $\beta_{i}=0$, we recover the compactness theorem of Cobos, Kühn and Schonbek (see [7, Theorem 4.8]).

3. Estimates for entropy numbers. When one of the $N$-tuples degenerates to a single Banach space, i.e. $A_{1}=\ldots=A_{N}=A$ or $B_{1}=\ldots=B_{N}=B$, we can improve Theorem 2.1 by estimating entropy numbers of the interpolated operator. 
Proposition 3.1. Let $\Pi=\overline{P_{1} \ldots P_{N}}$ be a convex polygon with vertices $P_{j}$, let $(\alpha, \beta) \in$ Int $\Pi$ and $1 \leq q \leq \infty$. For any Banach $N$-tuple $\bar{A}=\left\{A_{1}, \ldots, A_{N}\right\}$, any Banach space $B$ and any operator $T: \bar{A} \rightarrow \bar{B}$, we have

(i) $e_{n_{1}+\cdots+n_{N}-N+1}\left(T: \bar{A}_{(\alpha, \beta), q ; J} \rightarrow B\right) \leq C_{1} N e_{n_{1}}\left(T_{1}\right)^{\theta_{1}} \cdots e_{n_{N}}\left(T_{N}\right)^{\theta_{N}}$,

(ii) $e_{n_{1}+\cdots+n_{N}-N+1}\left(T: \bar{A}_{(\alpha, \beta), q ; K} \rightarrow B\right) \leq C_{2} N \max _{\{i, j, k\} \in \mathcal{P}_{(\alpha, \beta)}}\left\{e_{n_{i}}\left(T_{i}\right)^{c_{i}} e_{n_{j}}\left(T_{j}\right)^{c_{j}} e_{n_{k}}\left(T_{k}\right)^{c_{k}}\right\}$.

Here $T_{i}=T_{\mid A_{i}}, i=1, \ldots, N, \bar{\theta}=\left(\theta_{1}, \ldots, \theta_{N}\right)$ are barycentric coordinates of $(\alpha, \beta), C_{1}$ is a constant depending only on $\bar{\theta}$, and $C_{2}$ is another constant that depends only on $\Pi$ and $(\alpha, \beta)$. $\Sigma(\bar{A})$ :

Proof. For $i=1, \ldots, N$, take any $k_{i}>e_{n_{i}}\left(T_{i}\right)$ and consider the following norm on

$$
\|\mid a\|=\inf \left\{k_{1}\left\|a_{1}\right\|+\ldots+k_{N}\left\|a_{N}\right\|: a=\sum_{i=1}^{N} a_{i} ; a_{i} \in A_{i}\right\} .
$$

Given any $a \in \bar{A}_{(\alpha, \beta), q ; J}$ with $\|a\|_{(\alpha, \beta), q ; J}<1$, by the Hahn-Banach theorem, we can find a bounded functional $f \in(\Sigma(\bar{A}))^{*}$ such that $f(a)=\|a\| \|$ and $\|f\|_{A_{i}} \leq k_{i}$ for $i=1, \ldots, N$. According to (2), the norm of the restriction of $f$ to $\bar{A}_{(\alpha, \beta), q ; J}$ satisfies

$$
\|f\|_{\left(\bar{A}_{(\alpha, \beta), q ; J}\right) *} \leq C_{1} k_{1}^{\theta_{1}} \cdots k_{N}^{\theta_{N}} .
$$

Hence

$$
\left\|\left|a\left\||=| f(a) \mid \leq C_{1} k_{1}^{\theta_{1}} \cdots k_{N}^{\theta_{N}}\right\| a \|_{(\alpha, \beta), q ; J}<C_{1} k_{1}^{\theta_{1}} \cdots k_{N}^{\theta_{N}} .\right.\right.
$$

It follows that there is a representation $a=\sum_{i=1}^{N} a_{i}$ of $a$ with $\left\|a_{i}\right\|_{A_{i}}$
$\leq C_{1} k_{1}^{\theta_{1}} \cdots k_{i}^{\theta_{i}-1} \cdots k_{N}^{\theta_{N}}, 1 \leq i \leq N$. Thus

$$
\frac{a_{i}}{C_{1} k_{1}^{\theta_{1}} \cdots k_{i}^{\theta_{i}-1} \cdots k_{N}^{\theta_{N}}} \in \mathcal{U}_{A_{i}} .
$$

By definition of entropy numbers, there exists $b_{1}^{i}, \ldots, b_{s_{i}}^{i}$ with $s_{i} \leq 2^{n_{i}-1}$ so that

$$
T\left(\mathcal{U}_{A_{i}}\right) \subset \bigcup_{j=1}^{s_{i}}\left\{b_{j}^{i}+k_{i} \mathcal{U}_{B}\right\}, 1 \leq i \leq N
$$

We can then choose $j_{i}$ in such a way that

$$
\left\|T\left(a_{i}\right)-C k_{1}^{\theta_{1}} \cdots k_{i}^{\theta_{i}-1} \cdots k_{N}^{\theta_{N}} b_{j_{i}}^{i}\right\|_{B} \leq C_{1} k_{1}^{\theta_{1}} \cdots k_{N}^{\theta_{N}},
$$

and so

$$
\left\|T(a)-\left(C_{1} k_{1}^{\theta_{1}-1} \cdots k_{N}^{\theta_{N}} b_{j_{1}}^{1}+\cdots+C_{1} k_{1}^{\theta_{1}} \cdots k_{N}^{\theta_{N}-1} b_{j_{N}}^{N}\right)\right\|_{B} \leqslant C_{1} N k_{1}^{\theta_{1}} \cdots k_{N}^{\theta_{N}} .
$$

This yields the result

$$
e_{n_{1}+\cdots+n_{N}-N+1}\left(T: \bar{A}_{(\alpha, \beta), q ; J} \rightarrow B\right) \leq C_{1} N e_{n_{1}}\left(T_{1}\right)^{\theta_{1}} \cdots e_{n_{N}}\left(T_{N}\right)^{\theta_{N}} .
$$


Inequality (ii) follows from similar arguments but now using (1) to estimate the norm of the restriction of $f$ to $\bar{A}_{(\alpha, \beta), q ; K}$.

REMARK 3.2. Inequality (i) does not hold for $K$-spaces, as we show next by means of an example.

Let $\Pi=\{(0,0),(1,0),(0,1),(1,1)\}$ be the unit square, let $\bar{A}=\left\{\ell_{\infty}^{n}, \ell_{\infty}, \ell_{\infty}, \ell_{\infty}\right\}$, $B=l_{\infty}$ and let $T$ be the identity operator.

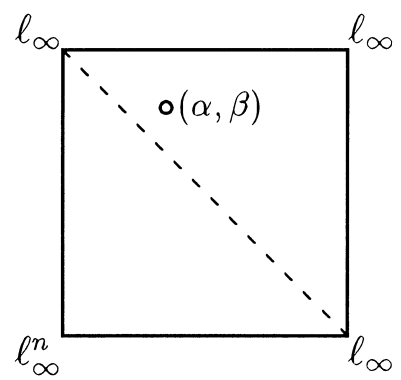

Figure 2.1

Choose $(\alpha, \beta)$ as in Fig. 2.1, i.e. in the interior of the triangle $\overline{(1,0),(0,1),(1,1)}$. Then, since $\ell_{\infty}^{n}$ is $n$-dimensional, $T: \ell_{\infty}^{n} \rightarrow \ell_{\infty}$ is compact. But $T:\left(\ell_{\infty}^{n}, \ell_{\infty}, \ell_{\infty}, \ell_{\infty}\right)_{(\alpha, \beta), q ; K} \rightarrow \ell_{\infty}$ fails to be compact, because, according to [4 Theorem 1.5], $\left(\ell_{\infty}^{n}, \ell_{\infty}, \ell_{\infty}, \ell_{\infty}\right)_{(\alpha, \beta), q ; K}=\ell_{\infty}$. In other words, $\lim _{n \rightarrow \infty} e_{n}\left(T: \bar{A}_{(\alpha, \beta), q ; K} \rightarrow B\right) \neq 0$ although $\lim _{n \rightarrow \infty} e_{n}\left(T: A_{1} \rightarrow B\right)=0$.

Next we turn our attention to the case when the operator starts from a degenerate $N$-tuple. This time the stronger result corresponds to $K$-spaces.

Proposition 3.3. Let $\Pi=\overline{P_{1} \ldots P_{N}}$ be a convex polygon with vertices $P_{j}$, let $(\alpha, \beta) \in \operatorname{Int} \Pi$ and $1 \leq q \leq \infty$. For any Banach $N$-tuple $\bar{B}=\left\{B_{1}, \ldots, B_{N}\right\}$, any Banach space $A$ and any operator $T: A \rightarrow \bar{B}$, we have

(i) $e_{n_{1}+\cdots+n_{N}-N+1}\left(T: A \rightarrow \bar{B}_{(\alpha, \beta), q ; K}\right) \leq 2 C_{1} N e_{n_{1}}\left(T_{1}\right)^{\theta_{1}} \cdots e_{n_{N}}\left(T_{N}\right)^{\theta_{N}}$,

(ii) $e_{n_{1}+\cdots+n_{N}-N+1}\left(T: A \rightarrow \bar{B}_{(\alpha, \beta), q ; J)} \leq 2 C_{2} N \max _{\{i, j, k\} \in \mathcal{P}_{(\alpha, \beta)}}\left\{e_{n_{i}}\left(T_{i}\right)^{c_{i}} e_{n_{j}}\left(T_{j}\right)^{c_{j}} e_{n_{k}}\left(T_{k}\right)^{c_{k}}\right\}\right.$.

Here $T_{i}=T: A \rightarrow B_{i}, i=1, \ldots, N, \bar{\theta}=\left(\theta_{1}, \ldots, \theta_{N}\right)$ are barycentric coordinates of $(\alpha, \beta), C_{1}$ is a constant depending only on $\bar{\theta}$, and $C_{2}$ is another constant that depends only on $\Pi$ and $(\alpha, \beta)$.

Proof. Given any $k_{i}>e_{n_{i}}\left(T_{i}\right)$, there are $\left\{y_{j_{i}}^{i}\right\}_{1 \leq j_{i} \leq s_{i}} \subseteq B_{i}$ with $s_{i} \leq 2^{n_{i}-1}$ and

$$
T\left(\mathcal{U}_{A}\right) \subset \bigcup_{j_{i}=1}^{s_{i}}\left\{y_{j_{i}}^{i}+k_{i} \mathcal{U}_{B_{i}}\right\}, 1 \leq i \leq N .
$$

Hence

$$
T\left(\mathcal{U}_{A}\right) \subset \bigcup_{\substack{1 \leq j_{1} \leq s_{1} \\ 1 \leq j_{N} \leq s_{N}}}\left(\bigcap_{i=1}^{N}\left\{y_{j_{i}}^{i}+k_{i} \mathcal{U}_{B_{i}}\right\}\right)
$$


Take $w_{\left(j_{1}, \ldots, j_{N}\right)} \in \bigcap_{i=1}^{N}\left\{y_{j_{i}}^{i}+k_{i} \mathcal{U}_{B_{i}}\right\}$ if the last set is non-empty. Then the number of the $w_{\left(j_{1}, \ldots, j_{N}\right)}$ is at most $2^{n_{1}+\cdots+n_{N}-N}$, and given any $a \in \mathcal{U}_{A}$ we can find $\left(j_{1}, \ldots, j_{N}\right)$ such that

$$
\left\|T a-w_{\left(j_{1}, \ldots, j_{N}\right)}\right\|_{(\alpha, \beta), q ; K} \leq C_{1} \prod_{i=1}^{N}\left\|T a-w_{\left(j_{1}, \ldots, j_{N}\right)}\right\|_{B_{i}}^{\theta_{i}} \leq 2 C_{1} \prod_{i=1}^{N} k_{i}^{\theta_{i}}
$$

where we have used (4) in the first inequality. This implies (i). Part (ii) follows by using (3) instead of (4).

REMARK 3.4. Let $\Pi=\{(0,0),(1,0),(0,1),(1,1)\}$ be the unit square, let $A=\ell_{1}(n)=\left\{\xi=\left(\xi_{n}\right):\|\xi\|_{\ell_{1}(n)}=\sum_{n=1}^{\infty} n\left|\xi_{n}\right|<\infty\right\}, \bar{B}=\left\{\ell_{1}, \ell_{1}(n), \ell_{1}(n), \ell_{1}(n)\right\}$ and let $T$ be the identity operator. Taking $(\alpha, \beta)$ as in Remark 3.2, it follows from [4, Theorem 1.5], that $\bar{B}_{(\alpha, \beta), q ; J}=\ell_{1}(n)$. Therefore $\lim _{n \rightarrow \infty} e_{n}\left(T: A \rightarrow \bar{B}_{(\alpha, \beta), q ; J} \neq 0\right.$ although $\lim _{n \rightarrow \infty} e_{n}\left(T: A \rightarrow B_{1}\right)=0$. Consequently, estimate (i) does not hold in general for $J$ spaces.

Remark 3.5. Proposition 3.1(ii) and Proposition 3.3(ii) yield Nikolova's results [10] mentioned in the Introduction, because $\lim _{n \rightarrow \infty} e_{n}(T)=\beta(T)$.

Compactness results in degenerate cases established by Cobos and Peetre in $[\mathbf{8}$, Section 4], and Cobos, Kühn and Schonbek [7, Proposition 4.5 and 4.6], follow also from Propositions 3.1 and 3.3.

ACKNOWLedgements. All three authors have been supported in part by DGICYT (PB94-0252).

\section{REFERENCES} 1976).

1. J. Bergh and J. Löfström, Interpolation spaces, an introduction (Springer-Verlag,

2. B. Carl and I. Stephani, Entropy, compactness and the approximation of operators (Cambridge University Press, 1990).

3. F. Cobos, On optimality of compactness results for interpolation methods associated to polygons, Indag. Math. 5 (1994), 397-401.

4. F. Cobos, P. Fernández-Martíinez and A. Martínez, On reiteration and the behaviour of weak compactness under certain interpolation methods, Collect. Math., to appear.

5. F. Cobos, P. Fernández-Martínez and A. Martínez, Interpolation of the measure of non-compactness by the real method, Studia. Math., to appear.

6. F. Cobos, P. Fernández-Martinez and T. Schonbek, Norm estimates for interpolation methods defined by means of polygons, J. Approx. Theory 80 (1995), 321-351.

7. F. Cobos, T. Kühn and T. Schonbek, One-sided compactness results for AronszajnGagliardo functors, J. Functional Analysis 106 (1992), 274-313.

8. F. Cobos and J. Peetre, Interpolation of compact operators: the multidimensional case, Proc. London Math. Soc. 63 (1991), 371-400.

9. D. E. Edmunds and W. D. Evans, Spectral theory and differential operators (Clarendon Press, Oxford, 1987). 
10. L. I. Nikolova, Some estimates of measure of non-compactness for operators acting in interpolation spaces - the multidimensional case, C.R. Acad. Bulg. Sci. 44 (1991), 5-8.

11. A. Pietsch, Operator ideals (North-Holland, Amsterdam, 1980).

12. M. F. Teixeira and D. E. Edmunds, Interpolation theory and measures of non-compactness, Math. Nachr. 104 (1981), 129-135.

13. H. Triebel, Interpolation theory, function spaces, differential operators (North-Holland Amsterdam, 1978). 